\title{
Melanoma Presenting as a Maxillary Sinus Mass: A Case Report
}

Kamal Gautam ${ }^{1}$, Sansar Tiwari² ${ }^{2}$, Suraj Shrestha ${ }^{2}$, Malati Dulal ${ }^{2}$, Bibek Shrestha ${ }^{2}$, Roman Dhital $^{3}$, and Usha Manandhar ${ }^{2}$

${ }^{1}$ Patan Academy of Health Sciences

${ }^{2}$ Tribhuvan University Institute of Medicine

${ }^{3}$ Tribhuvan University Teaching Hospital

August 26, 2021

\begin{abstract}
Maxillary sinus melanoma is a rare mucosal melanoma difficult to diagnose in the absence of pigmentation. Intranasal masses presenting with the features of occult malignancy and rapid progression should always be investigated in the line of melanoma irrespective of pigmentation. The histopathological and immunohistochemical examination helps to confirm the diagnosis.
\end{abstract}

\section{Melanoma Presenting as a Maxillary Sinus Mass: A Case Report}

Kamal Gautam, ${ }^{1}$ Sansar Babu Tiwari, ${ }^{2}$ Suraj Shrestha,${ }^{3}$ Prabin Gaire, ${ }^{2}$ Malati Dulal,${ }^{4}$ Bibek Man Shrestha, ${ }^{3}$ Roman Dhital, ${ }^{5}$ Usha Manandhar, ${ }^{2}$

Author affiliations:

${ }^{1}$ Oxford University Clinical Research Unit, Patan Academy of Health Sciences, Lagankhel, Kathmandu

${ }^{2}$ Department of Pathology, Tribhuvan University Teaching Hospital, Maharajgunj Medical Campus, Kathmandu, Nepal

${ }^{3}$ Maharajgunj Medical Campus, Institute of Medicine, Kathmandu, Nepal

${ }^{4}$ Department of ENT, Tribhuvan University Teaching Hospital, Maharajgunj Medical Campus, Kathmandu, Nepal

${ }^{5}$ Nepal National Hospital, Kathmandu, Nepal

Corresponding Author: Sansar Babu Tiwari, Department of Pathology, Tribhuvan University Teaching Hospital, Institute of Medicine, Kathmandu, Nepal, Email: sansartiwari@gmail.com Phone: +9779849331506 .

\section{KEY CLINICAL MESSAGE}

Maxillary sinus melanoma is a rare mucosal melanoma difficult to diagnose in the absence of pigmentation. Intranasal masses presenting with the features of occult malignancy and rapid progression should always be investigated in the line of melanoma irrespective of pigmentation. The histopathological and immunohistochemical examination helps to confirm the diagnosis.

Keywords: Intranasal melanoma; maxillary sinus; radiotherapy.

INTRODUCTION

Mucosal melanoma is a malignant neoplasm arising from melanocytes in the mucosa. Sinonasal melanoma accounts for $1 \%$ of all the melanomas encountered and $4 \%$ of all the sinonasal tumors. ${ }^{1}$ It is an uncommon 
finding with a reported incidence of 0.3 cases per million people per year but it accounts for approximately $25 \%$ of all melanoma in the Asian population. ${ }^{2}$ Most patients present with sinonasal congestion and epistaxis. It is highly aggressive due to its rich vascularity and tends to metastasize. It poses a diagnostic challenge as around $20 \%$ are multifocal and around $40 \%$ are amelanotic. ${ }^{2}$

Here we report a case of a 33-year-old female who presented with right nasal cavity mass and features of nasal obstruction for one and half months which on immunohistochemical examination showed melanoma.

\section{CASE REPORT}

A 33-year-old non-smoker female presented to the outpatient department with right nasal obstruction for one and half months, which was initially partial, gradually progressed to complete along with visualization of mass in the right nasal cavity. She also complained of blood-tinged right nasal discharge and right hemifacial pain for the same duration. The patient has lost 5 kilograms over 2 months and does not have any family history of malignancy.

On examination of the nose, externally, the right nasofacial groove was obliterated and on nasal vestibular examination, greyish-white mass was visualized occupying the whole of the right nasal cavity with the lowermost part of mass reaching just up to the nostril. Also, the right eyeball showed non-axial proptosis. (Figure 1)

On blood investigation, hemoglobin level was $11 \mathrm{~g} / \mathrm{dl}$, and all other parameters were within normal limits. CT scan of nose and paranasal sinuses showed enhancing soft tissue opacification in the right maxillary sinus, ethmoid and frontal sinus extending through the eroded and widened osteo-meatal complex medially into the nasal cavity with multiple hyperdensities. Also, the mass was eroding the maxillary walls, right lamina papyracea, cribriform plate, and extending up to the right orbital cavity and right infratemporal fossa. (Figure 2). Punch biopsy was done under local anesthesia which yielded multiple pieces of greyish white tissues without melanin pigmentation. Histopathological examination showed sheets of singly dispersed tumor cells with scant cytoplasm, coarse chromatin, and prominent nucleoli without necrosis along with focal cartilaginous changes.

Immunohistochemical studies showed strong and diffuse HMB-45 and weak vimentin positivity. The tumor cells were negative for S100, CD45, cytokeratin, and desmin. (Figure 3) With these features, a diagnosis of malignant melanoma was made. The patient is currently undergoing radiotherapy. Surgical removal of the tumor is planned, however; the patient is reluctant to undergo surgery due to economic constraints and is undergoing radiotherapy. She is planned to be evaluated after 3 months.

\section{DISCUSSION}

Mucosal melanoma of the head and neck region is an uncommon entity and accounts for $1 \%$ of all melanomas without sex predilection. ${ }^{3}$ The entity is common in the seventh decade of life. Most of the mucosal melanomas arise from the melanocytes in the lining epithelium. Sinonasal melanomas have a possible association with melanocytosis which is seen in uveal melanomas. The nasal cavity is the most common site followed by the septum and nasopharynx. However, the maxillary sinus is the least encountered site. ${ }^{4}$ Our patient is an adult female of age 33 and had a right maxillary sinus mass.

Although formaldehyde exposure and tobacco smoking are considered to play a role in pathogenesis, the specific etiological factors are not well elucidated. ${ }^{5,6}$ Unlike cutaneous and uveal melanomas, molecular studies of mucosal melanomas show higher rates of KIT mutations, followed by NRAS and BRAF mutations. Our patient is a non-smoker and does not have any exposure to formaldehyde.

Most of the mucosal melanomas occur in the occult site and present late in the course of the disease. ${ }^{7}$ However, they are of aggressive behavior in comparison to their cutaneous and uveal counterparts resulting in rapid progression of symptoms and early metastasis. Our patient presented with features of nasal obstruction and blood-stained nasal discharge for one and half months. The mass is infiltrating the surrounding bones and adjacent structures in the CT scan. 
Macroscopically mucosal melanomas show polypoidal growth of black-colored and friable to greyish and firm mass. Amelanotic presentation is more common in sinonasal type (20-25\%) compared to cutaneous type (1.8-8.1\%). ${ }^{8}$ Our patient had greyish discoloration of the mass originating from the right maxillary antrum and protruding into the right nasal cavity.

Imaging including CT of local bones and MRI can be helpful to evaluate tumor extension. CT with 3D facial reconstruction is especially helpful in patients planned for facial reconstruction. A spontaneous highintensity signal on T1 with a low-intensity signal on T2 is a typical brain and facial MRI finding. MRI also helps to differentiate between melanoma and paranasal sinus fluid retention, delineate the borders of invasion and detect any brain metastases. Furthermore, chest, abdomen, and pelvis CT and PET scan help stage the tumor and thereby helps management. ${ }^{9}$ Our patient did not have evidence of metastasis in the head and neck region. Abdominal and chest CT scans were not done.

Histologically, it is a challenging task to diagnose mucosal melanomas in absence of melanin pigmentation. Immunohistochemical stain helps to reach the diagnosis. There are different morphological types of tumor cells ranging from spindle to epithelioid to plasmacytoid. Immunohistochemically, S100 and HMB-45 are positive even in tumor cells lacking melanin pigment ${ }^{10}$. However, cytokeratin is absent. Our patient had epithelioid-type cells on microscopy with tumor cells showing positivity with HMB-45 stain.

Sometimes the sinonasal melanoma could be a presentation of the metastatic spectrum of the melanoma elsewhere which changes the staging of the disease. American Joint Committee on Cancer staging manual eight editions has mentioned that all head and neck melanoma should be kept in the T3-T4 category irrespective of the size of the mass. ${ }^{3}$ The overall prognosis is decreased if there is any distant metastasis and advanced age. Our patient is an adult female and did not have any metastasis.

Wide surgical resection is widely accepted as the first-line treatment of head and neck mucosal melanomas until we can resect the tumor with a negative margin. The role of adjuvant radiation therapy can be considered if the tumor can be resected completely. As mucosal melanomas are poorly radiosensitive, radiotherapy is reserved for tumors with positive surgical margins, local recurrence, locally advanced tumors, and for patients who refuse surgery or sometimes as palliative therapy. In patients with surgical treatment failure, metastatic disease, or palliation, chemotherapy can be used. The use of immunotherapy as a monotherapy or adjunct therapy in combination with other treatments is still under evaluation. Considering the poor outcome and financial status, our patient refused to undergo any sort of surgery and is under radiotherapy. She is planned for evaluation after completion of radiotherapy.

Despite aggressive therapy, the prognosis of mucosal melanomas is poor with 5-year survival rates of 12 to $30 \% .{ }^{11}$ Risk of local recurrence ranges from $37-54 \%$ and metastases are found in around $50 \%$ of cases. ${ }^{12}$ The head and neck melanomas recur within a median time of 6 to 12 months. ${ }^{13}$ Negative resection margin is the most important prognostic factor. The prognosis of the disease depends on obstructive symptoms, advanced age, tumor size, vascular invasion, location, high mitotic count, marked cellular pleomorphism, and regional and distant metastasis. Other poor prognostic factors include advanced stage, old age, tumor size greater than $3-4 \mathrm{~cm} .{ }^{14}$

\section{CONCLUSION}

Primary malignant melanoma of the nose is an extremely rare entity and it must be differentiated from the other tumors of the nose and paranasal sinuses. Due to its highly aggressive nature and its tendency to metastasize early, it should be diagnosed at the earliest and surgically resected promptly to increase the chances of survival. Hence, patients with minor nasal symptoms should also be assessed thoroughly to rule out any neoplastic etiologies.

\section{ACKNOWLEDGEMENTS}

We gratefully acknowledge the work of members of our hospital and the patient.

\section{ETHICAL APPROVAL AND CONSENT TO PARTICIPATE}


Not applicable.

\section{CONSENT FOR PUBLICATION}

Written informed consent was obtained from the patient before the submission of the report. The signed Institutional Consent Form is on file.

\section{CONFLICTS OF INTEREST STATEMENT}

None

FUNDING/FINANCIAL OUTCOMES

None

\section{AUTHOR'S CONTRIBUTION}

DATA AVAILABILITY: The data that support the findings of this study are available from the corresponding author upon reasonable request.

\section{REFERENCES}

1. Ascierto PA, Accorona R, Botti G, et al. Mucosal melanoma of the head and neck. Crit Rev Oncol Hematol 2017; 112: 136-152. 2017/03/23. DOI: 10.1016/j.critrevonc.2017.01.019.

2. Wahid NW, Meghji S and Barnes M. Nasal mucosal melanoma. The Lancet Oncology 2019; 20: e284. DOI: 10.1016/S1470-2045(19)30179-2.

3. El-Naggar AK, Chan JKC, Rubin Grandis J, et al. WHO classification of head and neck tumours . 2017.

4. Bachar G, Loh KS, O'Sullivan B, et al. Mucosal melanomas of the head and neck: experience of the Princess Margaret Hospital. Head $\&$ neck 2008; 30: 1325-1331. 2008/08/16. DOI: 10.1002/hed.20878.

5. Benevenuto de Andrade BA, Piña AR, León JE, et al. Primary nasal mucosal melanoma in Brazil: clinicopathologic and immunohistochemical study of 12 patients. Annals of diagnostic pathology 2012; 16: 344-349. 2012/03/20. DOI: 10.1016/j.anndiagpath.2012.02.001.

6. Mihajlovic M, Vlajkovic S, Jovanovic P, et al. Primary mucosal melanomas: a comprehensive review.International journal of clinical and experimental pathology2012; 5: 739-753. 2012/10/17.

7. Baderca F, Vincze D, Balica N, et al. Mucosal melanomas in the elderly: challenging cases and review of the literature. Clinical interventions in aging 2014; 9: 929-937. 2014/06/25. DOI: 10.2147/cia.S64361.

8. Seetharamu N, Ott PA and Pavlick AC. Mucosal melanomas: a case-based review of the literature. The oncologist 2010; 15: 772-781. 2010/06/24. DOI: 10.1634/theoncologist.2010-0067.

9. Gilain L, Houette A, Montalban A, et al. Mucosal melanoma of the nasal cavity and paranasal sinuses.European Annals of Otorhinolaryngology, Head and Neck Diseases2014; 131: 365-369. DOI: https://doi.org/10.1016/j.anorl.2013.11.004.

10. Notani K, Shindoh M, Yamazaki Y, et al. Amelanotic malignant melanomas of the oral mucosa. The British journal of oral \& maxillofacial surgery 2002; 40: 195-200. 2002/06/11. DOI: 10.1054/bjom.2001.0713.

11. Patel SG, Prasad ML, Escrig M, et al. Primary mucosal malignant melanoma of the head and neck. Head \& neck 2002; 24: 247-257. 2002/03/14. DOI: 10.1002/hed.10019.

12. Liétin B, Montalban A, Louvrier C, et al. Sinonasal mucosal melanomas. European Annals of Otorhinolaryngology, Head and Neck Diseases 2010; 127: 70-76. DOI: https://doi.org/10.1016/j.anorl.2010.04.006.

13. Manolidis S and Donald PJ. Malignant mucosal melanoma of the head and neck: review of the literature and report of 14 patients. Cancer 1997; 80: 1373-1386. 1997/10/24 21:28. DOI: 10.1002/(sici)10970142(19971015)80:8<1373::aid-cncr3>3.0.co;2-g. 
14. Pol Ong YB and Doh Jeing Y. Sinonasal malignant melanoma: a case report. 2019 2019; 5: 3 \%J International Journal of Otorhinolaryngology and Head and Neck Surgery. Mucosal melanoma, Sinonasal tumour, Unilateral nasal tumour, Malignant melanoma 2019-04-26. DOI: 10.18203/issn.2454-5929.ijohns20191757.

\section{LEGENDS}

Figure 1 : Right nasolabial fold obliteration and mass reaching up to the right nostril.

Figure 2 : CT Nose and PNS demonstrating soft tissue opacification in the right maxillary sinus, ethmoid and frontal sinus along with the erosion of surrounding bony structures.

Figure 3 : a). Sheets of dispersed tumor cells with a scant amount of cytoplasm and coarse chromatin. b) Tumor cells show moderate diffuse cytoplasmic HMB-45 positivity, c) weak vimentin positivity and d) negative CD45 immunostaining.

\section{FIGURES}

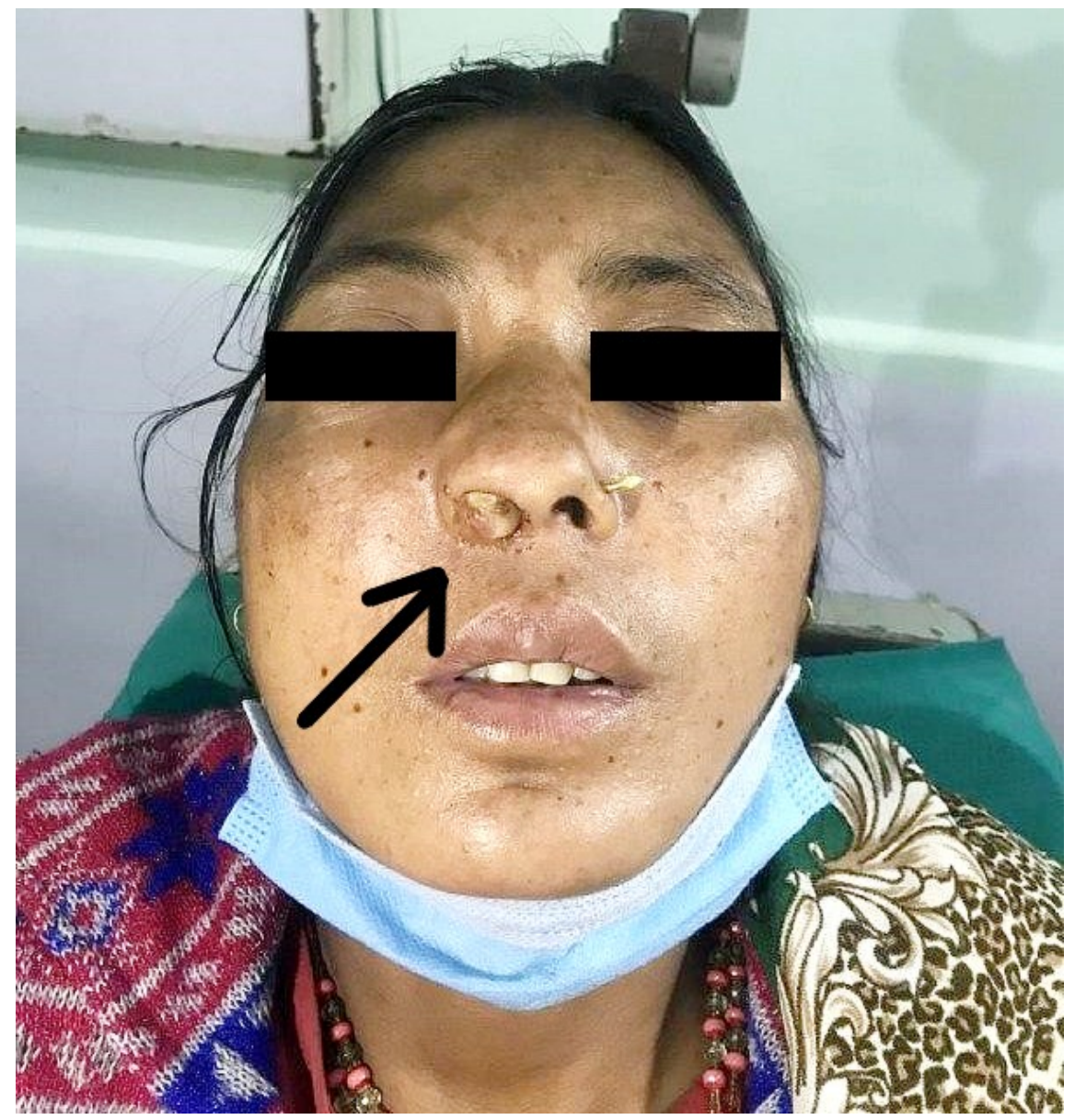

Figure 1 : Right nasolabial fold obliteration and mass reaching up to the right nostril. 


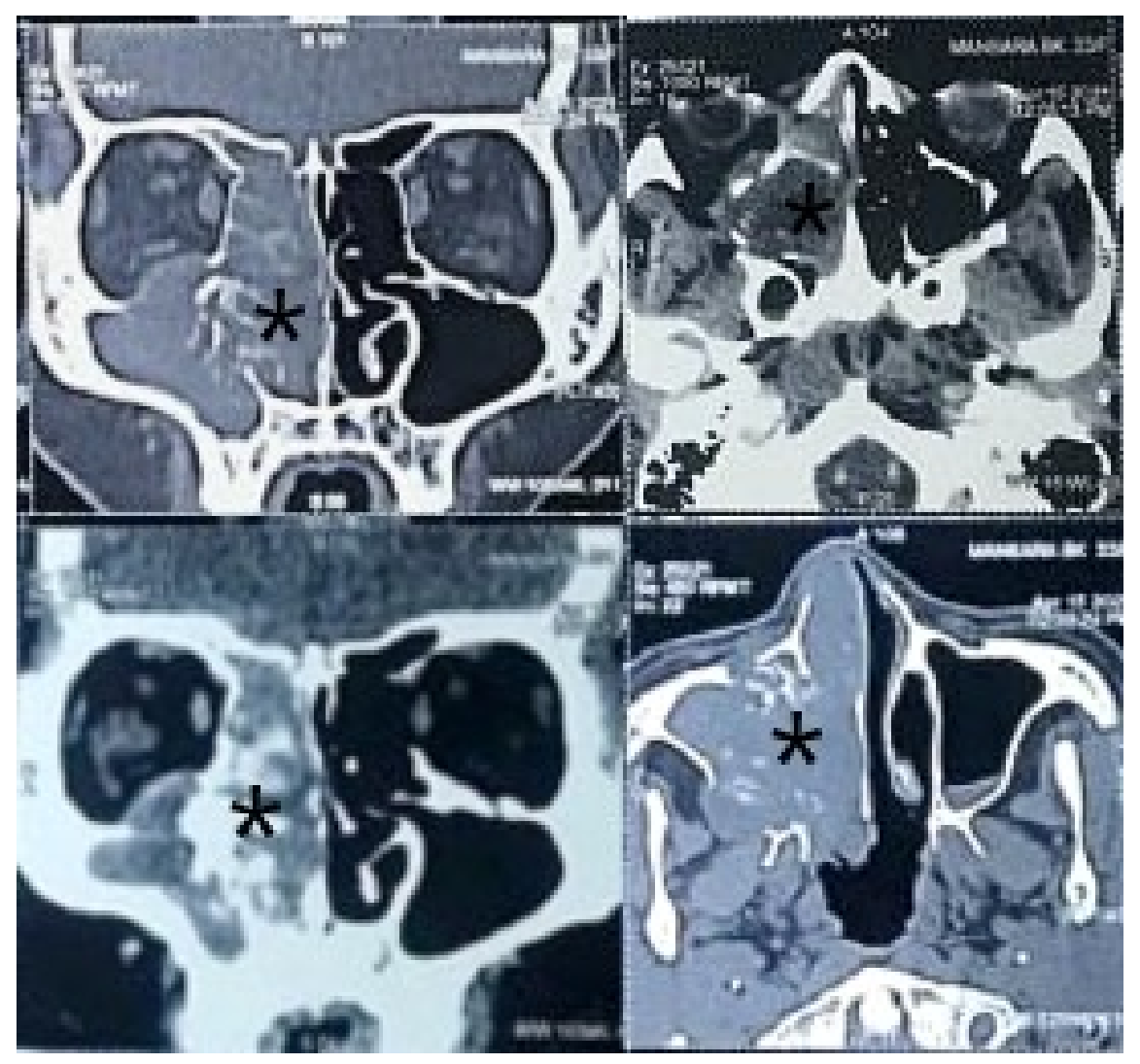

Figure 2 : CT Nose and PNS demonstrating soft tissue opacification in the right maxillary sinus, ethmoid and frontal sinus along with the erosion of surrounding bony structures.

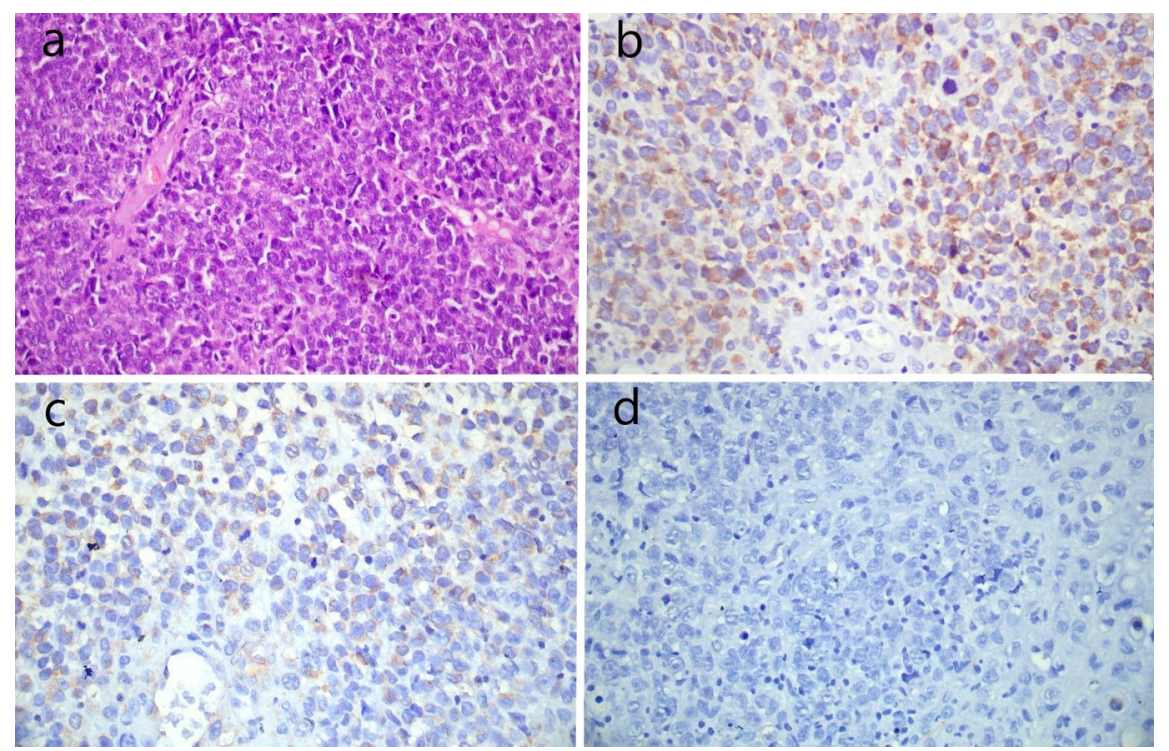

Figure 3: a). Sheets of dispersed tumor cells with a scant amount of cytoplasm and coarse chromatin. b) Tumor cells show moderate diffuse cytoplasmic HMB-45 positivity, c) weak vimentin positivity and d) 
negative CD45 immunostaining.

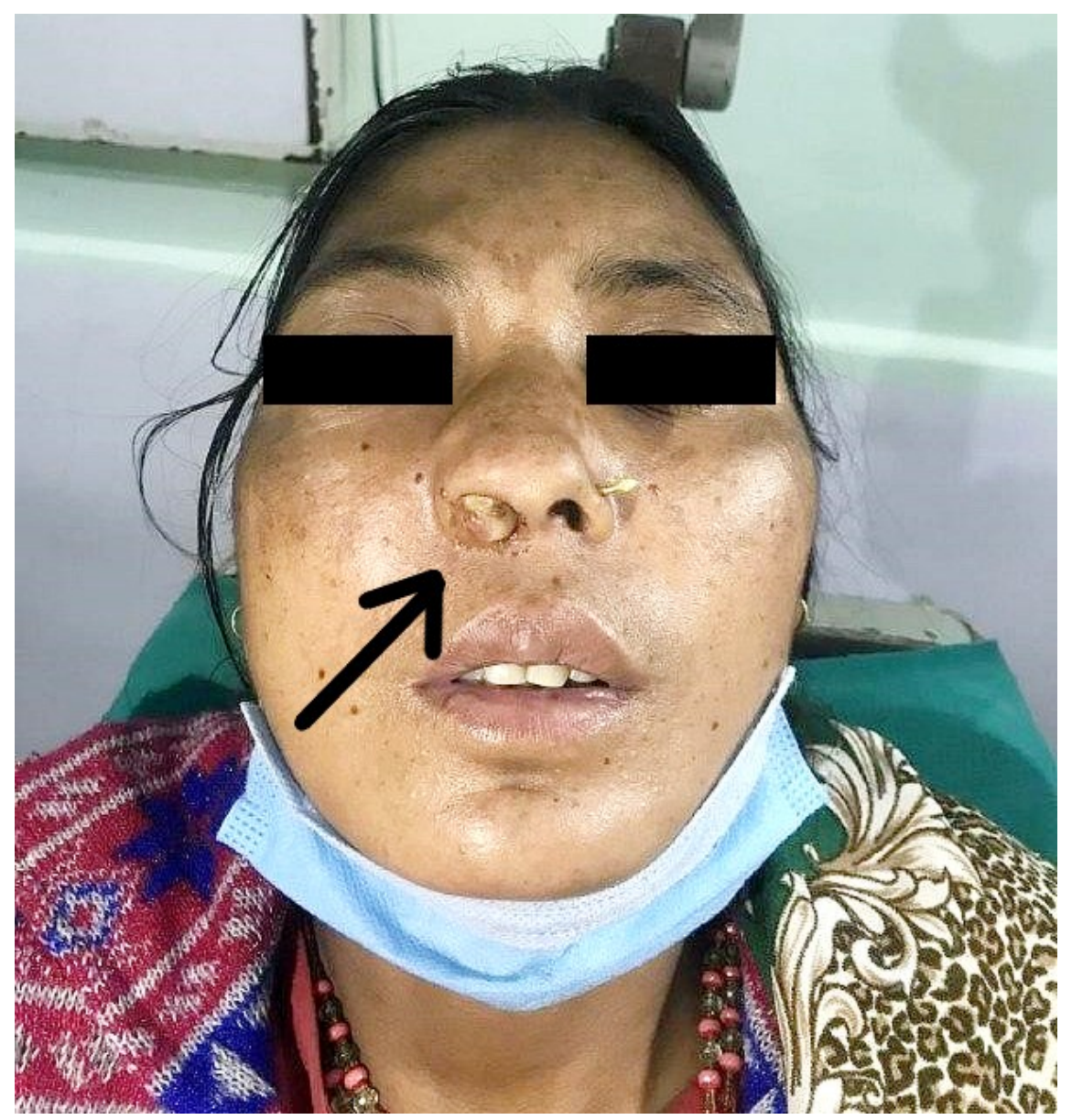



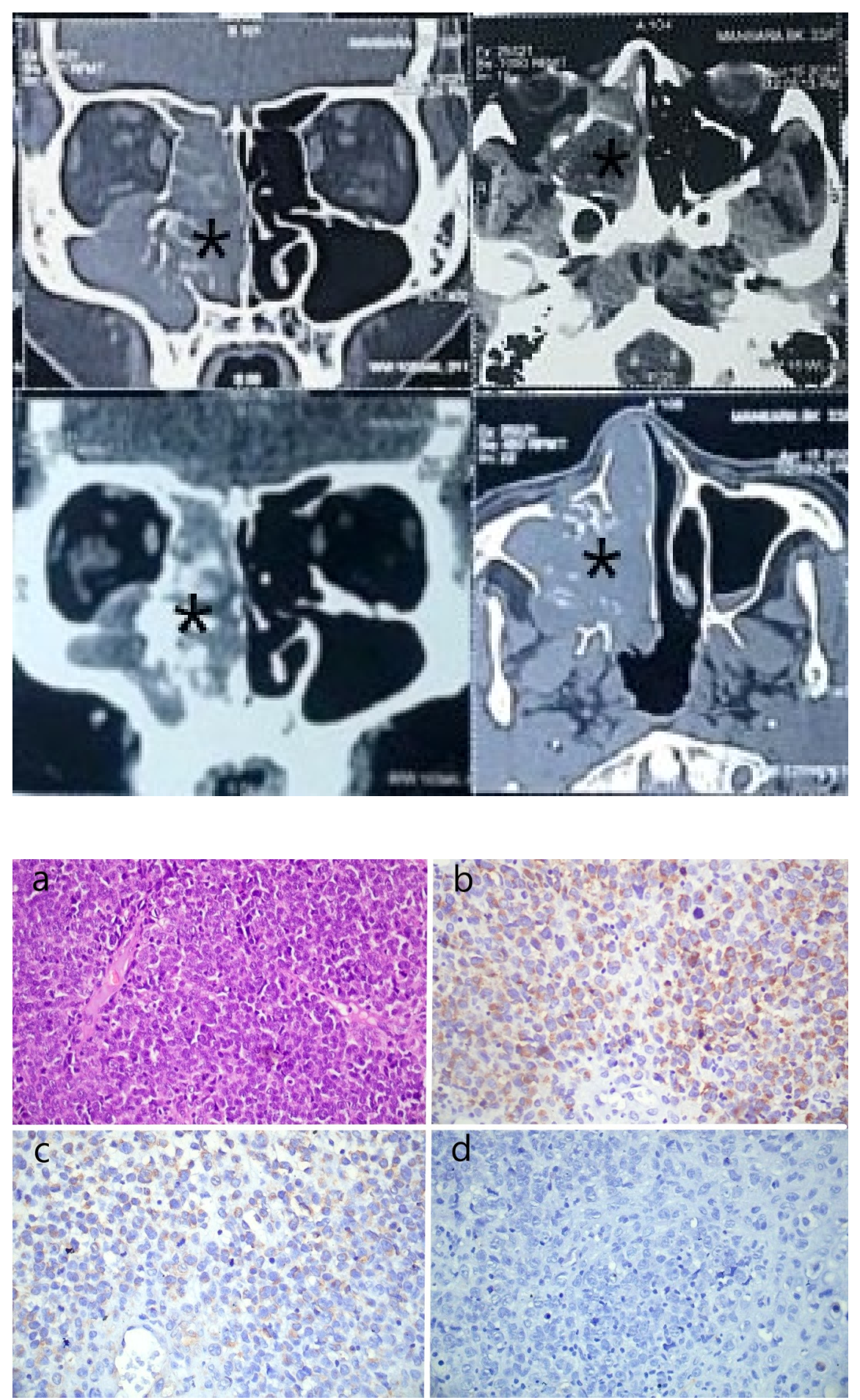